\title{
Vacuum polarization induced coupling between Maxwell and Kalb-Ramond fields
}

\author{
N. D. Hari Dass* and K. V. Shajesh ${ }^{\dagger}$ \\ Institute of Mathematical Sciences, C. I. T. Campus, Taramani, Chennai- 600 113, India.
}

\begin{abstract}
We present here a manifestly gauge invariant calculation of vacuum polarization to fermions in the presence of a constant Maxwell and a constant Kalb-Ramond field in four dimensions. The formalism is a generalisation of the one used by Schwinger in his famous paper on gauge invariance and vacuum polarization. We get an explicit expression for the vacuum polarization induced effective Lagrangian for a constant Maxwell field interacting with a constant Kalb-Ramond field. In the weak field limit we get the coupling between the Maxwell field and the Kalb-Ramond field to be $(\tilde{H} . \tilde{F})^{2}$, where $\tilde{H}_{\mu}=\frac{1}{3 !} \epsilon_{\mu \alpha \beta \lambda} H^{\alpha \beta \lambda}$ and $\tilde{F}$ is the dual of $F_{\mu \nu}$.
\end{abstract}

\section{INTRODUCTION}

Consider the interaction involving the fermion field, the Maxwell field and the KalbRamond field. Let us approximate the Maxwell field and the Kalb-Ramond field to be classical background fields. We address to the following question: what will be the induced effective Lagrangian for the classical background (consisting of the Maxwell field and the Kalb-Ramond field) because of the vacuum polarization of the quantised fermion field? This task is achieved by integrating out the fermion field.

\footnotetext{
*email : dass@imsc.ernet.in

†email : kvshajesh@yahoo.com
} 
The evaluation of the vacuum polarization induced effective Lagrangian for a classical background field interacting with a quantum field originated in the works of W. Heisenberg and H. Euler [1] and V. Weisskopf [2] before the advent of QED. An explicit expression within QED for the vacuum polarization induced effective Lagrangian for the classical background electromagnetic field interacting with the quantised fermion field was evaluated by Julian Schwinger in [3].

Quantum electrodynamics is a $U(1)$ gauge theory in four dimensional Minkowski spacetime. It involves a 1-form gauge potential $A_{\mu}(x)$ and a matter field $\psi(x)$ acting as the source of $A_{\mu}(x)$. In QED the source of $A_{\mu}(x)$ is a point particle. The Lagrangian for the 1-form quantum electrodynamics

$$
\mathcal{L}=-\frac{1}{4} F_{\mu \nu} F^{\mu \nu}+\bar{\psi}(x)(i \not \partial-e \not A-m) \psi(x)
$$

is constructed by demanding invariance of the Lagrangian under the $U(1)$ gauge transformations

$$
\begin{aligned}
A_{\mu}(x) & \rightarrow A_{\mu}(x)+\partial_{\mu} \Lambda^{A}(x) \\
\psi(x) & \rightarrow \exp \left\{i e \Lambda^{A}(x)\right\} \psi(x)
\end{aligned}
$$

where $\Lambda^{A}(x)$ is an arbitrary 0 -form field. $F_{\mu \nu}(x)=\partial_{\mu} A_{\nu}-\partial_{\nu} A_{\mu}$ is the 2 -form antisymmetric field tensor constructed from the 1-form gauge potential $A_{\mu}(x)$.Requirements of renormalisability pose additional requirements and eqn (1) is a minimal choice.

The p-form quantum electrodynamics [4 6] is a $U(1)$ gauge theory constructed out of the p-form gauge potential $A_{\mu_{1} \ldots \mu_{p}}$. As the gauge transformation parameter in such cases is a $p-1$-form, the analog of eqn(2) makes sense only if $\psi$ represents a $p-1$-dimensional extended object. In particular 2-form quantum electrodynamics is a $U(1)$ gauge theory which involves a 2-form gauge potential $B_{\mu \nu}(x)$ and a string matter field $\psi[x(\sigma)]$ for the source of $B_{\mu \nu}(x)$. The Lagrangian for 2-form quantum electrodynamics

$$
\mathcal{L}=-\frac{1}{12} H_{\mu \nu \lambda} H^{\mu \nu \lambda}+\bar{\psi}[x(\sigma)] \Gamma_{\mu \nu}\left(i \frac{\delta}{\delta \Omega_{\mu \nu}}-\mu A^{\mu \nu}\right) \psi[x(\sigma)]
$$


is constructed by demanding invariance under the $U(1)$ gauge transformations

$$
\begin{aligned}
B_{\mu \nu}(x) & \rightarrow B_{\mu \nu}(x)+\partial_{\mu} \Lambda_{\nu}^{B}(x)-\partial_{\nu} \Lambda_{\mu}^{B}(x) \\
\psi[x(\sigma)] & \rightarrow \exp \left\{i \mu \int_{\sigma} \Lambda_{\mu}^{B}(x) d x^{\mu}\right\} \psi[x(\sigma)]
\end{aligned}
$$

where $\Lambda_{\mu}^{B}(x)$ is an arbitrary 1-form field. $H_{\mu \nu \lambda}(x)=\partial_{\mu} B_{\nu \lambda}+\partial_{\nu} B_{\lambda \mu}+\partial_{\lambda} B_{\mu \nu}$ is the 3-form totally antisymmetric field tensor constructed from the 2-form antisymmetric gauge potential $B_{\mu \nu}(x) . H_{\mu \nu \lambda}(x)$ is popularly called the Kalb-Ramond field. The functional derivative $\frac{\delta}{\delta \Omega_{\mu \nu}}$ captures the variation of $\psi[x(\sigma)]$ due to an infinitesimal change of the string configuration which sweeps a surface element $d \Omega_{\mu \nu}$.

Kalb-Ramond fields play important roles in string theories and field theories [7,8]. In $d=4$ the Kalb-Ramond field is dual to the derivative of a spinless field $\phi$ which can be interpreted as an axion. We shall not elaborate too much on the importance of the Kalb-Ramond field here. We believe that the exercise we have carried out in this paper is interesting in its own right.

As the system represented by eqns $(3,4)$ is too complicated since it involves string theory,in this paper we have investigated the simpler problem of the interaction of a point-like fermion field with Maxwell and Kalb-Ramond fields in four dimensional Minkowski spacetime. This is only possible if $\psi$ carries no Kalb-Ramond charge. Nevertheless, we show that a nontrivial effective Lagrangian between Maxwell and Kalb-Ramond fields emerges. The simplest Lagrangian we can construct by demanding invariance under the $U(1)$ gauge transformations

$$
\begin{aligned}
B_{\mu \nu}(x) & \rightarrow B_{\mu \nu}(x)+\partial_{\mu} \Lambda_{\nu}^{B}(x)-\partial_{\nu} \Lambda_{\mu}^{B}(x) \\
A_{\mu}(x) & \rightarrow A_{\mu}(x)+\partial_{\mu} \Lambda^{A}(x) \\
\psi(x) & \rightarrow \exp \left\{i e \Lambda^{A}(x)\right\} \psi(x)
\end{aligned}
$$

is

$$
\mathcal{L}=-\frac{1}{4} F_{\mu \nu} F^{\mu \nu}-\frac{1}{12} H_{\mu \nu \lambda} H^{\mu \nu \lambda}+\bar{\psi}\left(i \not \partial-e / A-\frac{1}{12} \frac{g}{m} \sigma_{\mu \nu \lambda} H^{\mu \nu \lambda}-m\right) \psi
$$


Such a Lagrangian could arise from string theories after compactification to four dimensional space-time.

Schwinger's calculation in [3] involves evaluation of the vacuum polarization induced effective Lagrangian for the case of 1-form quantum electrodynamics. In this work we generalize this to the Lagrangian given in eqn. (6). We evaluate an explicit expression for the vacuum polarization induced effective Lagrangian for the classical background (consisting of Maxwell and Kalb-Ramond fields) interacting with the quantised fermion field. We present the paper exactly along the lines of Schwinger's paper. As can be seen subsequently, there are important differences between our case and that of Schwinger. Due to these reasons our calculation could give some insight if one attempts to generalize Schwinger's calculation for other circumstances like the non abelian gauge theories. An important check over our calculations is that at every stage we are able to reproduce Schwinger's results for QED by taking the limit $g \rightarrow 0$.

In section [1] we formulate the methodology used by Schwinger in [3] for a general background field. We integrate out the fermion field. As in [3] the determination of the vacuum polarization induced effective Lagrangian reduces to the evaluation of the trace of the exponential of a suitable operator. Schwinger interprets the exponential of the operator as an evolution operator describing the dynamics of a "particle" with a "Hamiltonian" evolving in "proper time".

In section III we exactly solve the evolution operator for the case of a constant Maxwell field and a constant Kalb-Ramond field. The Hamiltonian under consideration can be understood as a generalization of Schwinger's case. There are additional complications in our case arising out of operators not commuting with each other.

The effective Lagrangian is determined by evaluating the trace of the evolution operator in both the coordinate index and the spinor index. This involves solving for the eigenvalues of the Maxwell field $F_{\mu \nu}$ in the coordinate index and of another operator $Q$ in the spinor index. The determination of the eigenvalues is simplified by constructing suitable polynomial equations which are satisfied by $F_{\mu \nu}$ and $Q$. The eigenvalues are conveniently expressed in 
terms of gauge and Lorentz invariant quantities.

We thus get an explicit expression for the vacuum polarization induced effective Lagrangian for the case of constant field strengths in terms of gauge invariant quantities. Renormalization of the two field strengths $F_{\mu \nu}$ and $H_{\mu \nu \lambda}$, and the two coupling constants $e$ and $g$ leads to a divergence free expression for the effective Lagrangian. For the case of weak field strengths we show that the leading order interaction between the Maxwell field and the Kalb-Ramond field is given by $(\tilde{H} . \tilde{F})^{2}$, where $\tilde{H}_{\mu}=\frac{1}{3 !} \epsilon_{\mu \alpha \beta \lambda} H^{\alpha \beta \lambda}$ and $\tilde{F}$ is the dual of $F_{\mu \nu}$.

\section{FORMULATION OF THE GENERAL PROBLEM}

In this section we formulate Schwinger's techniques for the case of a fermion field interacting with external Maxwell and Kalb-Ramond fields. In the next section we obtain the exact solutions for the case of constant external fields.

In eqn. (6) we have seen that the Lagrangian for a spin $\frac{1}{2}$ fermion field interacting with a Kalb-Ramond field and a Maxwell field is given by

$$
\mathcal{L}[H, F, \psi]=-\frac{1}{4} F_{\mu \nu} F^{\mu \nu}-\frac{1}{12} H_{\mu \nu \lambda} H^{\mu \nu \lambda}+\bar{\psi}\left(i \not \partial-e / A-\frac{1}{12} \frac{g}{m} \sigma_{\mu \nu \lambda} H^{\mu \nu \lambda}-m\right) \psi,
$$

where $\psi(x)$ is the fermion field. Our aim is to evaluate the effective Lagrangian arising from fermionic vacuum polarisation at one loop level. This amounts to integrating out the contribution of the fermion field. Using the above prescription we can evaluate the effective Lagrangian $\mathcal{L}_{e f f}[F, H]$ from the expression

$$
e^{i \int d^{4} x \mathcal{L}_{e f f}[F, H]}=\frac{\int \mathcal{D} \psi \int \mathcal{D} \bar{\psi} e^{i \int d^{4} x\left[-\frac{1}{4} F_{\mu \nu} F^{\mu \nu}-\frac{1}{12} H_{\mu \nu \lambda} H^{\mu \nu \lambda}+\bar{\psi}\left(i \not \partial-e A-\frac{1}{12} \frac{g}{m} \sigma_{\mu \nu \lambda} H^{\mu \nu \lambda}-m\right) \psi\right]}}{\int \mathcal{D} \psi \int \mathcal{D} \bar{\psi} e^{i \int d^{4} x \bar{\psi}(i \not \partial-m) \psi}}
$$

This can be thought of as the path integral realisation of Schwinger's approach. Using the Gaussian integral formula for the anticommuting fermion field

$$
\int \mathcal{D} \psi \int \mathcal{D} \bar{\psi} e^{-\int d^{4} x \bar{\psi} M \psi}=\text { const } \operatorname{Det} M
$$

in eqn. (8) we have 


$$
e^{i \int d^{4} x \mathcal{L}_{e f f}[F, H]}=e^{i \int d^{4} x\left[-\frac{1}{4} F^{2}-\frac{1}{12} H^{2}\right]}\left[\frac{\operatorname{Det}\left(i \not \partial-e \not A-\frac{1}{12} \frac{g}{m} \sigma_{\mu \nu \lambda} H^{\mu \nu \lambda}-m\right)}{\operatorname{Det}(i \not \partial-m)}\right] .
$$

The determinant Det is over both the coordinate and the spinor index. The notation we shall use is, Det(or Tr) to denote determinant(or trace) over both the coordinate and spinor index, and $\operatorname{det}($ or tr) to denote determinant(or trace) over only the spinor index. The determinant of an operator $M$ remains invariant under a similarity transformation $S$, i.e. Det $M=\operatorname{Det}\left(S M S^{-1}\right)$. Choosing $S$ to be the charge conjugation matrix $C$ we can write

$$
\text { Det } M=\left\{\operatorname{Det} M \cdot \operatorname{Det}\left(C M C^{-1}\right)\right\}^{\frac{1}{2}}=\left\{\operatorname{Det}\left(M C M C^{-1}\right)\right\}^{\frac{1}{2}} \text {, }
$$

Using eqn. (11) in eqn. (10) we get

$$
e^{i \int d^{4} x \mathcal{L}_{e f f}[F, H]}=e^{i \int d^{4} x\left[-\frac{1}{4} F^{2}-\frac{1}{12} H^{2}\right]}\left[\frac{\operatorname{Det} \mathcal{H}}{\operatorname{Det} \mathcal{H}_{0}}\right]^{\frac{1}{2}}
$$

where

$$
\begin{aligned}
\mathcal{H} & =\left(i \not \partial-e \not A-\frac{1}{12} \frac{g}{m} \sigma_{\mu \nu \lambda} H^{\mu \nu \lambda}-m\right)\left(-i \not \partial+e \not A-\frac{1}{12} \frac{g}{m} \sigma_{\mu \nu \lambda} H^{\mu \nu \lambda}-m\right) \\
\mathcal{H}_{0} & =(i \not \partial-m)(-i \not \partial-m) .
\end{aligned}
$$

We have used the fact that the gamma matrices transform under the charge conjugation matrix as $C \gamma_{\mu} C^{-1}=-\gamma_{\mu}^{T}$. Further, the identity Det $M=\exp [\operatorname{Tr} \ln M]$ and eqn. (12) gives

$$
\int d^{4} x \mathcal{L}_{e f f}[F, H]=\int d^{4} x\left[-\frac{1}{4} F^{2}-\frac{1}{12} H^{2}\right]-\frac{i}{2} \operatorname{Tr}\left[(\ln \mathcal{H})-\left(\ln \mathcal{H}_{0}\right)\right]
$$

Using the integral representation

$$
\ln M=-\int_{0}^{\infty} \frac{d s}{s} e^{-i M s}
$$

in eqn. (15) we have

$$
\int d^{4} x \mathcal{L}_{e f f}[F, H]=\int d^{4} x\left[-\frac{1}{4} F^{2}-\frac{1}{12} H^{2}\right]+\frac{i}{2} \int_{0}^{\infty} \frac{d s}{s}\left\{\left[\operatorname{Tr} e^{-i \mathcal{H} s}\right]-\left[\operatorname{Tr} e^{-i \mathcal{H}_{0} s}\right]\right\} .
$$

Convergence requires that $m$ be interpreted as $m-i \epsilon$. Eqn(16) has to be regularised carefully and this will be discussed later. 
Let us denote

$$
U\left(x^{\prime \prime}, x^{\prime} ; s\right) \equiv<x^{\prime \prime}\left|e^{-i \mathcal{H} s}\right| x^{\prime}>
$$

Following Schwinger, we shall treat $s$ as a sort of time variable called "proper time" by him and use a Heisenberg-like picture, in which the operators evolve in time.If $A(0)$ is an operator at $s=0$, the proper time dependence of the opertor is defined by

$$
A(s) \equiv e^{i \mathcal{H} s} A(0) e^{-i \mathcal{H} s}
$$

and

$$
<x^{\prime \prime}(s)\left|\equiv<x^{\prime \prime}\right| e^{-i \mathcal{H} s}
$$

It then follows that

$$
\begin{gathered}
<x^{\prime \prime}(s)\left|x(s)=<x^{\prime \prime}(s)\right| x^{\prime \prime} \\
x(0)\left|x^{\prime}>=x^{\prime}\right| x^{\prime}>
\end{gathered}
$$

Thus the problem of determining the effective Lagrangian reduces to the evaluation of $U(x(s), x(0) ; s) \equiv e^{-i \mathcal{H} s} . U(x(s), x(0) ; s)$ can be interpreted as the operator describing the development of a quantum mechanical system governed by the "Hamiltonian" $\mathcal{H}$ in the "time" $s$ from a state $\mid x^{\prime}(0)>$ to another state $\mid x^{\prime \prime}(s)>$. Care must be taken to distinguish the operator valued $U(x(s), x(0) ; s)$ from the c-number valued function $U\left(x^{\prime \prime}, x^{\prime} ; s\right)$.

It is useful to choose $x(s)$ and $x(0)$ as independent variables. If we were doing classical mechanics, we would have interpreted $U(x(s), x(0) ; s)$ as the generating function for the canonical transformation

$$
\begin{aligned}
& x_{\mu}(s)=x_{\mu}(x(0), p(0), s) \\
& p_{\mu}(s)=p_{\mu}(x(0), p(0), s) .
\end{aligned}
$$

and solved for $U(x(s), x(0) ; s)$ using the Hamilton Jacobi equations [9]. Equations corresponding to the Hamilton Jacobi equations for the quantum mechanical case are [10,11] 


$$
\begin{aligned}
\mathcal{H} U(x(s), x(0) ; s) & =i \frac{\partial}{\partial s} U(x(s), x(0) ; s) \\
\pi_{\mu}(0) U\left(x^{\prime \prime}, x^{\prime} ; s\right) & =\left\{-i \frac{\partial}{\partial x_{\mu}^{\prime}}-e A_{\mu}\left(x^{\prime}\right)\right\} U\left(x^{\prime \prime}, x^{\prime} ; s\right) \\
\pi_{\mu}(s) U\left(x^{\prime \prime}, x^{\prime} ; s\right) & =\left\{+i \frac{\partial}{\partial x_{\mu}^{\prime \prime}}-e A_{\mu}\left(x^{\prime \prime}\right)\right\} U\left(x^{\prime \prime}, x^{\prime} ; s\right)
\end{aligned}
$$

with the initial condition

$$
\lim _{s \rightarrow 0} U\left(x^{\prime \prime}, x^{\prime} ; s\right)=\delta^{(4)}\left(x^{\prime \prime}-x^{\prime}\right)
$$

where $\pi_{\mu}(s)=p_{\mu}(s)-e A_{\mu}(s)$. Thus evaluation of $U\left(x^{\prime \prime}, x^{\prime} ; s\right)$ will involve solving the first order differential equations (23-25) with the initial condition in eqn. (26) in conjunction with the Heisenberg equations of motion for the operators $x_{\mu}(s)$ and $p_{\mu}(s)$ given by

$$
\begin{aligned}
& \frac{d x_{\mu}(s)}{d s}=-i\left[x_{\mu}(s), \mathcal{H}\right] \\
& \frac{d \pi_{\mu}(s)}{d s}=-i\left[\pi_{\mu}(s), \mathcal{H}\right]
\end{aligned}
$$

The basic strategy is to try and express $\mathcal{H}$ in terms of $x_{\mu}(s), x_{\mu}(0)$ such that $x_{\mu}(s)$ always appear to the left and $x_{\mu}(0)$ always on the right.Then all operators can be traded for cnumbers and one gets a first order differential equation which is in principle solvable. It is not always possible to solve for $U\left(x^{\prime \prime}, x^{\prime} ; s\right)$ exactly. A general series solution for $U\left(x^{\prime \prime}, x^{\prime} ; s\right)$ can be got using the method by DeWitt [12]. In the next section we shall exactly solve the above first order differential equations for the case when $H$ and $F$ are constant fields. In the general case after solving for $U\left(x^{\prime \prime}, x^{\prime} ; s\right)$ using eqn. (23), eqn. (24) and eqn. (25) we can write

$$
\operatorname{Tr}\left[e^{-i \mathcal{H} s}\right]=\operatorname{Tr}\left\{U\left(x^{\prime \prime}, x^{\prime} ; s\right)\right\}=\operatorname{tr} \int d^{4} x\left\{U\left(x^{\prime \prime}, x^{\prime} ; s\right)\right\}_{x^{\prime \prime}=x^{\prime}=x}
$$

Using eqn. (28) in eqn. (17) we have

$$
\mathcal{L}_{e f f}[F, H]=-\frac{1}{4} F^{2}-\frac{1}{12} H^{2}+\frac{i}{2} \operatorname{tr} \int_{0}^{\infty} \frac{d s}{s}\left\{U\left(x^{\prime \prime}, x^{\prime} ; s\right)-U_{0}\left(x^{\prime \prime}, x^{\prime} ; s\right)\right\}_{x^{\prime \prime}=x^{\prime}=x} .
$$




\section{CONSTANT FIELDS}

In this section we shall evaluate an exact expression for the effective Lagrangian given in eqn. (29) for the case of constant $F$ and $H$ fields 13. This is achieved by solving for $U\left(x^{\prime \prime}, x^{\prime} ; s\right)$ using eqn. (23), (24), (25) and (27). The divergent terms in the effective Lagrangian are absorbed by suitable renormalization of the field strengths $F_{\mu \nu}$ and $H_{\mu \nu \lambda}$ and the couplings $e$ and $g$. Making a series expansion of the effective Lagrangian for the case of weak fields we get the leading order interaction between the Maxwell field and the Kalb-Ramond field to be $(\tilde{H} \cdot \tilde{F})^{2}$ analogous to the Euler-Heisenberg effective Lagrangian for electromagnetic fields.

For the case of constant $F$ and $H$ fields the expression for $\mathcal{H}$ in eqn. (13) simplifies to

$$
\mathcal{H}=-\pi_{\mu} \pi^{\mu}-N_{\mu} \pi^{\mu}-\frac{1}{4} N_{\mu} N^{\mu}+m^{2}+Q
$$

where

$$
\pi_{\mu}=i \partial_{\mu}-e A_{\mu}, \quad N_{\mu}=-i \frac{g}{m} \tilde{H}_{\mu} \gamma_{5} \quad \text { and } \quad Q=\frac{1}{2} e \sigma_{\mu \nu} F^{\mu \nu}+i g \tilde{H}_{\mu} \gamma_{5} \gamma^{\mu}
$$

where $\tilde{H}_{\mu}$ is the dual tensor corresponding to $H_{\mu \nu \lambda}$ and is given by $\tilde{H}_{\mu}=\frac{1}{3 !} \epsilon_{\mu \alpha \beta \lambda} H^{\alpha \beta \lambda}$. It should be noted that $N_{\mu}$ and $Q$ do not commute with each other. This is the additional complication we referred to earlier.The above simplification assumes that $B_{\mu \nu}(x)$ and $A_{\mu}(x)$ are classical (not quantised) fields. We also assume that the corresponding tensor fields $H_{\mu \nu \lambda}(x)$ and $F_{\mu \nu}(x)$ are constants in space and time. The above simplification also uses the identities,

$$
\sigma_{\alpha \beta \lambda}=i \epsilon_{\alpha \beta \lambda \mu} \gamma_{5} \gamma^{\mu} \quad \text { and } \quad\left[\sigma_{\alpha \beta \lambda}, \gamma_{\sigma}\right]=2 i \epsilon_{\alpha \beta \lambda \sigma} \gamma_{5}
$$

For the case of constant $F$ and $H$ fields the Heisenberg equations of motion for $x(s)$ and $\pi(s)$ in eqn. (27) simplifies to

$$
\begin{aligned}
& \frac{d x_{\mu}(s)}{d s}=2 \pi_{\mu}+N_{\mu} \\
& \frac{d \pi_{\mu}(s)}{d s}=e F_{\mu \alpha}\left\{2 \pi^{\alpha}(s)+N^{\alpha}\right\} .
\end{aligned}
$$


Using eqn. (33) we can solve for $\pi_{\mu}(s)$ and $\pi_{\mu}(0)$ in terms of $x_{\mu}(s)$ and $x_{\mu}(0)$ to get

$$
\begin{aligned}
& \vec{\pi}(0)=\left(\frac{e \hat{F}}{e^{2 e \hat{F} s}-1}\right) \cdot[\vec{x}(s)-\vec{x}(0)]-\frac{1}{2} \vec{N} \\
& \vec{\pi}(s)=\left(\frac{e \hat{F}}{1-e^{-2 e \hat{F} s}}\right) \cdot[\vec{x}(s)-\vec{x}(0)]-\frac{1}{2} \vec{N}
\end{aligned}
$$

where we have used the symbolic notation, ' $\vec{a}$ ' (vector) to denote $a_{\mu}$ and ' $\hat{A}$ ' (matrix) to denote $A_{\mu \nu}$. Observe that in this notation $a_{\mu} a^{\mu}$ will be denoted by $\vec{a}^{T} \cdot \vec{a}$. It is important to note that the transpose of the above expressions will not be the same, because of the fact that $\hat{F}^{T}=-\hat{F}$ (i.e. $\left.F_{\mu \nu}=-F_{\nu \mu}\right)$. Using eqn. (33) we can also solve for $x_{\mu}(s)$ and $\pi_{\mu}(s)$ in terms of $x_{\mu}(0)$ and $\pi_{\mu}(0)$ to get

$$
\begin{aligned}
& \vec{x}(s)=\vec{x}(0)+\left(\frac{e^{2 e \hat{F} s}-1}{e \hat{F}}\right) \cdot \vec{\pi}(0)+\frac{1}{2}\left(\frac{e^{2 e \hat{F} s}-1}{e \hat{F}}\right) \cdot \vec{N} \\
& \vec{\pi}(s)=e^{2 e \hat{F} s} \cdot \vec{\pi}(0)+\frac{1}{2} e^{2 e \hat{F} s} \cdot \vec{N}-\frac{1}{2} \vec{N}
\end{aligned}
$$

Using eqn. (34) in eqn. (30) we get

$$
\mathcal{H}=-\left[x_{\mu}(s)-x_{\mu}(0)\right] K^{\mu \nu}\left[x_{\nu}(s)-x_{\nu}(0)\right]+m^{2}+Q
$$

where

$$
\hat{K}=\frac{1}{4}\left(\frac{e \hat{F}}{\sinh (e \hat{F} s)}\right)^{2}
$$

Rearranging the order of the operators in eqn. (36) so that they are ordered in time we get $\mathcal{H}=-x_{\mu}(s) K^{\mu \nu} x_{\nu}(s)+2 x_{\mu}(s) K^{\mu \nu} x_{\nu}(0)-x_{\mu}(0) K^{\mu \nu} x_{\nu}(0)+m^{2}+Q-\frac{i}{2} \operatorname{tr}\left[\frac{e \hat{F}}{\tanh (e \hat{F} s)}\right]$

where we have used eqn. (35) to get

$$
x_{\mu}(0) K^{\mu \nu} x_{\nu}(s)-x_{\mu}(s) K^{\mu \nu} x_{\nu}(0)=-\frac{i}{2} \operatorname{tr}\left[\frac{e \hat{F}}{\tanh (e \hat{F} s)}\right]
$$

Using eqn. (38) and eqn. (34) the first order differential equations for $U\left(x^{\prime \prime}, x^{\prime} ; s\right)$ in eqn. (23), (24) and (25) takes the form

$$
\left\{-\left(x^{\prime \prime}-x^{\prime}\right)_{\mu} K^{\mu \nu}\left(x^{\prime \prime}-x^{\prime}\right)_{\nu}+m^{2}+Q-\frac{i}{2} \operatorname{tr}\left[\frac{e \hat{F}}{\tanh (e \hat{F} s)}\right]\right\} U\left(x^{\prime \prime}, x^{\prime} ; s\right)=i \frac{\partial}{\partial s} U\left(x^{\prime \prime}, x^{\prime} ; s\right)
$$




$$
\begin{gathered}
\left\{\left[\frac{e \hat{F}}{e^{2 e \hat{F} s}-1}\right]^{\mu \nu}\left(x^{\prime \prime}-x^{\prime}\right)_{\nu}-\frac{1}{2} N^{\mu}\right\} U\left(x^{\prime \prime}, x^{\prime} ; s\right)=\left\{-i \frac{\partial}{\partial x_{\mu}^{\prime}}-e A^{\mu}\left(x^{\prime}\right)\right\} U\left(x^{\prime \prime}, x^{\prime} ; s\right) \\
\left\{\left[\frac{e \hat{F}}{1-e^{-2 e \hat{F} s}}\right]^{\mu \nu}\left(x^{\prime \prime}-x^{\prime}\right)_{\nu}-\frac{1}{2} N^{\mu}\right\} U\left(x^{\prime \prime}, x^{\prime} ; s\right)=\left\{+i \frac{\partial}{\partial x_{\mu}^{\prime \prime}}-e A^{\mu}\left(x^{\prime \prime}\right)\right\} U\left(x^{\prime \prime}, x^{\prime} ; s\right)
\end{gathered}
$$

with the initial condition in eqn. (26). Integrating eqn. (40) with respect to $s$ on both sides we get

$$
\begin{aligned}
U\left(x^{\prime \prime}, x^{\prime} ; s\right)=C\left(x^{\prime \prime}, x^{\prime}\right) \frac{1}{s^{2}} e^{-i m^{2} s} e^{-i Q s}\left[\operatorname{det} \frac{e \hat{F} s}{\sinh (e \hat{F} s)}\right]^{\frac{1}{2}} \\
\exp \left\{-i \frac{1}{4 s}\left[\frac{e \hat{F} s}{\tanh (e \hat{F} s)}\right]^{\mu \nu}\left(x^{\prime \prime}-x^{\prime}\right)_{\mu}\left(x^{\prime \prime}-x^{\prime}\right)_{\nu}\right\}
\end{aligned}
$$

where $C\left(x^{\prime \prime}, x^{\prime}\right)$ is the integration constant which has no explicit dependence on $s$. Using the above expression for $U\left(x^{\prime \prime}, x^{\prime} ; s\right)$ in eqn. (41) and (42) we get the differential equations for $C\left(x^{\prime \prime}, x^{\prime}\right)$ to be

$$
\begin{aligned}
& \left\{-i \frac{\partial}{\partial x_{\mu}^{\prime}}-e A^{\mu}\left(x^{\prime}\right)+\frac{1}{2} N^{\mu}+\frac{1}{2} e F^{\mu \nu}\left(x^{\prime \prime}-x^{\prime}\right)_{\nu}\right\} C\left(x^{\prime \prime}, x^{\prime}\right)=0 \\
& \left\{+i \frac{\partial}{\partial x_{\mu}^{\prime \prime}}-e A^{\mu}\left(x^{\prime \prime}\right)+\frac{1}{2} N^{\mu}-\frac{1}{2} e F^{\mu \nu}\left(x^{\prime \prime}-x^{\prime}\right)_{\nu}\right\} C\left(x^{\prime \prime}, x^{\prime}\right)=0
\end{aligned}
$$

Integrating eqn. (44) and eqn. (45) we get

$$
C\left(x^{\prime \prime}, x^{\prime}\right)=C_{0} \phi\left(x^{\prime \prime}, x^{\prime}\right)
$$

where $C_{0}$ is the integration constant and

$$
\phi\left(x^{\prime \prime}, x^{\prime}\right)=\exp \left[-i e \int_{x^{\prime}}^{x^{\prime \prime}} d t_{\mu}\left\{A^{\mu}(t)+N^{\mu}\right\}\right] .
$$

$C_{0}$ is determined using the initial condition in eqn. (26) to give

$$
C_{0}=-i \frac{1}{16 \pi^{2}}
$$

where we have used the representation for the delta function

$$
\delta\left(x-x^{\prime}\right)=\lim _{\sigma \rightarrow 0} \frac{1}{\sqrt{2 \pi \sigma}} \exp \left\{-\frac{\left(x-x^{\prime}\right)^{2}}{2 \sigma}\right\} .
$$

Thus we have 


$$
\begin{aligned}
U\left(x^{\prime \prime}, x^{\prime} ; s\right)=-i \frac{1}{16 \pi^{2}} \phi\left(x^{\prime \prime}, x^{\prime}\right) & \frac{1}{s^{2}} e^{-i m^{2} s} e^{-i Q s}\left[\operatorname{det} \frac{e \hat{F} s}{\sinh (e \hat{F} s)}\right]^{\frac{1}{2}} \\
& \exp \left\{-i \frac{1}{4 s}\left[\frac{e \hat{F} s}{\tanh (e \hat{F} s)}\right]^{\mu \nu}\left(x^{\prime \prime}-x^{\prime}\right)_{\mu}\left(x^{\prime \prime}-x^{\prime}\right)_{\nu}\right\}
\end{aligned}
$$

It should be emphasised that $\phi\left(x^{\prime \prime}, x^{\prime}\right)$ does-not commute with the rest of the $U_{0}\left(x^{\prime \prime}, x^{\prime} ; s\right)$. Due to this reason it is necessary that $\phi\left(x^{\prime \prime}, x^{\prime}\right)$ operate on to the left of $U_{0}\left(x^{\prime \prime}, x^{\prime} ; s\right)$. We can evaluate $U_{0}\left(x^{\prime \prime}, x^{\prime} ; s\right)=e^{-i \mathcal{H}_{0} s}$ independently or by taking the limit $F$ and $H$ going to zero in eqn. (50) to get

$$
U_{0}\left(x^{\prime \prime}, x^{\prime} ; s\right)=-i \frac{1}{16 \pi^{2}} \frac{1}{s^{2}} e^{-i m^{2} s} \mathbf{1} \exp \left\{-i \frac{1}{4 s}\left(x^{\prime \prime}-x^{\prime}\right)_{\mu}\left(x^{\prime \prime}-x^{\prime}\right)_{\nu}\right\}
$$

where 1 is the unit matrix in the spinor space. Using eqn. (50) and eqn. (51) we have

$$
\begin{aligned}
& \operatorname{Tr}\left[U\left(x^{\prime \prime}, x^{\prime} ; s\right)\right]=-i \frac{1}{16 \pi^{2}} \int d^{4} x \frac{1}{s^{2}} e^{-i m^{2} s}\left\{\operatorname{det} \frac{e \hat{F} s}{\sinh (e \hat{F} s)}\right\}^{\frac{1}{2}}\left\{\operatorname{tr} e^{-i Q s}\right\} \\
& \operatorname{Tr}\left[U_{0}\left(x^{\prime \prime}, x^{\prime} ; s\right)\right]=-i \frac{1}{16 \pi^{2}} \int d^{4} x \frac{1}{s^{2}} e^{-i m^{2} s} 4
\end{aligned}
$$

where 4 comes from the trace over the spinor index. Using eqn. (52) and eqn. (53) in eqn. (29) we get

$\mathcal{L}_{\text {eff }}[F, H]=-\frac{1}{4} F^{2}-\frac{1}{12} H^{2}+\frac{1}{32 \pi^{2}} \int_{0}^{\infty} \frac{d s}{s^{3}} e^{-i m^{2} s}\left[\left\{\operatorname{det} \frac{e \hat{F} s}{\sinh (e \hat{F} s)}\right\}^{\frac{1}{2}}\left\{\operatorname{tr} e^{-i Q s}\right\}-4\right]$

Thus our problem reduces to determination of the eigenvalues of $\hat{F}$ and $Q$. Note that $\hat{F}$ is a matrix in space time and $Q$ is a matrix in the spinor index. We shall determine the eigenvalues of $\hat{F}$ and $Q$ in terms of the Lorentz invariants

$$
\begin{aligned}
\mathcal{F} & =\frac{1}{4} F_{\mu \nu} F^{\mu \nu} & \mathcal{G} & =\frac{1}{4} F_{\mu \nu} \tilde{F}^{\mu \nu} \\
\mathcal{H} & =\frac{1}{12} H_{\mu \nu \lambda} H^{\mu \nu \lambda} & \mathcal{I}^{2} & =\frac{1}{48}\left(H_{\lambda \mu \nu} F^{\mu \nu}\right)\left(H^{\lambda \alpha \beta} F_{\alpha \beta}\right)=-\frac{1}{12}\left(\tilde{H}_{\mu} \tilde{F}^{\mu \lambda}\right)\left(\tilde{F}_{\lambda \nu} \tilde{H}^{\nu}\right)
\end{aligned}
$$

where $\tilde{F}^{\mu \nu}$ is the dual tensor corresponding to $F^{\mu \nu}$ and is given by $\tilde{F}^{\mu \nu}=\frac{1}{2} \epsilon^{\mu \nu \alpha \beta} F_{\alpha \beta}$.

Eigen values of $\hat{F}$ is determined [3] by observing that $\hat{F}$ satisfies the identity

$$
\hat{F}^{4}+2 \mathcal{F} \hat{F}^{2}-\mathcal{G}^{2}=0
$$


This can be verified using the identities

$$
F_{\mu \lambda} \tilde{F}^{\lambda \nu}=-\delta_{\mu}{ }^{\nu} \mathcal{G} \quad \text { and } \quad \tilde{F}_{\mu \lambda} \tilde{F}^{\lambda \nu}-F_{\mu \lambda} F^{\lambda \nu}=2 \delta_{\mu}{ }^{\nu} \mathcal{F}
$$

Using eqn. (56) we have the eigenvalues of $\hat{F}$ to be $\pm \lambda_{1}$ and $\pm \lambda_{2}$ where

$$
\begin{aligned}
& \lambda_{1}=\frac{i}{\sqrt{2}}\left[\{\mathcal{F}+i \mathcal{G}\}^{\frac{1}{2}}+\{\mathcal{F}-i \mathcal{G}\}^{\frac{1}{2}}\right] \\
& \lambda_{2}=\frac{i}{\sqrt{2}}\left[\{\mathcal{F}+i \mathcal{G}\}^{\frac{1}{2}}-\{\mathcal{F}-i \mathcal{G}\}^{\frac{1}{2}}\right]
\end{aligned}
$$

Thus we have

$$
\left\{\operatorname{det} \frac{e \hat{F} s}{\sinh (e \hat{F} s)}\right\}^{\frac{1}{2}}=\frac{\left(e \lambda_{1} s\right)}{\sinh \left(e \lambda_{1} s\right)} \frac{\left(e \lambda_{2} s\right)}{\sinh \left(e \lambda_{2} s\right)}
$$

We shall now determine the eigenvalues of $Q$. Beginning from the expression for $Q$ in eqn. (31) we have

$$
Q^{2}=(a-d) 1+i c_{\alpha} \gamma^{\alpha}+i b \gamma_{5}
$$

and

$$
Q^{4}=\left\{(a-d)^{2}-b^{2}-c^{2}\right\} 1+2 i(a-d) c_{\alpha} \gamma^{\alpha}+2 i(a-d) b \gamma_{5}
$$

where

$$
\begin{array}{rlrl}
a & =2 e^{2} \mathcal{F} & b & =2 e^{2} \mathcal{G} \\
d & =2 g^{2} \mathcal{H} & c_{\alpha} & =4 \sqrt{3} e g \mathcal{I}_{\alpha}
\end{array}
$$

In arriving at eqn. (61) and eqn. (62) we have used the identities

$$
\left\{\sigma_{\mu \nu}, \sigma_{\alpha \beta}\right\}=2\left(g_{\mu \alpha} g_{\nu \beta}-g_{\mu \beta} g_{\nu \alpha}\right) 1+2 i \epsilon_{\mu \nu \alpha \beta} \gamma_{5} \quad \text { and } \quad\left\{\sigma_{\mu \nu}, \gamma_{5} \gamma_{\alpha}\right\}=-2 \epsilon_{\mu \nu \alpha \sigma} \gamma^{\sigma}
$$

and

$$
\tilde{H}_{\mu} \tilde{H}^{\mu}=-\frac{1}{6} H_{\mu \nu \lambda} H^{\mu \nu \lambda} \quad \text { and } \quad \tilde{H}^{\mu} \tilde{F}_{\mu \lambda}=-\frac{1}{2} H_{\lambda \alpha \beta} F^{\alpha \beta}
$$

Using eqn. (61) and eqn. (62) it is easy to verify that $Q$ satisfies the relation 


$$
Q^{4}-2(a-d) Q^{2}+\left\{(a-d)^{2}+b^{2}+c^{2}\right\}=0
$$

Thus the eigenvalues of $Q$ are $\pm e q_{1}$ and $\pm e q_{2}$, where

$$
\begin{aligned}
& e q_{1}=\sqrt{2}\left\{\left(e^{2} \mathcal{F}-g^{2} \mathcal{H}\right)+i \sqrt{e^{4} \mathcal{G}^{2}+12 e^{2} g^{2} \mathcal{I}^{2}}\right\}^{\frac{1}{2}} \\
& e q_{2}=\sqrt{2}\left\{\left(e^{2} \mathcal{F}-g^{2} \mathcal{H}\right)-i \sqrt{e^{4} \mathcal{G}^{2}+12 e^{2} g^{2} \mathcal{I}^{2}}\right\}^{\frac{1}{2}}
\end{aligned}
$$

Using eqn. (67) we have

$$
\left\{\operatorname{tr} e^{-i Q s}\right\}=4 \cosh \left(e \chi_{1} s\right) \cosh \left(e \chi_{2} s\right)
$$

where

$$
\begin{aligned}
& e \chi_{1}=\frac{i}{\sqrt{2}}\left[\left\{\left(e^{2} \mathcal{F}-g^{2} \mathcal{H}\right)+i \sqrt{e^{4} \mathcal{G}^{2}+12 e^{2} g^{2} \mathcal{I}^{2}}\right\}^{\frac{1}{2}}+\left\{\left(e^{2} \mathcal{F}-g^{2} \mathcal{H}\right)-i \sqrt{e^{4} \mathcal{G}^{2}+12 e^{2} g^{2} \mathcal{I}^{2}}\right\}^{\frac{1}{2}}\right] \\
& e \chi_{2}=\frac{i}{\sqrt{2}}\left[\left\{\left(e^{2} \mathcal{F}-g^{2} \mathcal{H}\right)+i \sqrt{e^{4} \mathcal{G}^{2}+12 e^{2} g^{2} \mathcal{I}^{2}}\right\}^{\frac{1}{2}}-\left\{\left(e^{2} \mathcal{F}-g^{2} \mathcal{H}\right)-i \sqrt{e^{4} \mathcal{G}^{2}+12 e^{2} g^{2} \mathcal{I}^{2}}\right\}^{\frac{1}{2}}\right]
\end{aligned}
$$

Observe that in the limit $g \rightarrow 0$ we have $\chi_{1} \rightarrow \lambda_{1}$ and $\chi_{2} \rightarrow \lambda_{2}$. Using eqn. (60) and eqn. (68) in eqn. (54) we have

$$
\mathcal{L}_{e f f}[F, H]=-\mathcal{F}-\mathcal{H}+\frac{1}{8 \pi^{2}} \int_{0}^{\infty} \frac{d s}{s^{3}} e^{-i m^{2} s}\left[\left(e \lambda_{1} s\right)\left(e \lambda_{2} s\right) \frac{\cosh \left(e \chi_{1} s\right)}{\sinh \left(e \lambda_{1} s\right)} \frac{\cosh \left(e \chi_{2} s\right)}{\sinh \left(e \lambda_{2} s\right)}-1\right]
$$

Expressing the above integral in terms of the contour running along the positive real axis (cf. eqn. (17) ) which is effectively got by substituting $s \rightarrow-i s$ in the integral, we get

$$
\mathcal{L}_{e f f}[F, H]=-\mathcal{F}-\mathcal{H}-\frac{1}{8 \pi^{2}} \int_{0}^{\infty} \frac{d s}{s^{3}} e^{-m^{2} s}\left[\left(e \lambda_{1} s\right)\left(e \lambda_{2} s\right) \frac{\cos \left(e \chi_{1} s\right)}{\sin \left(e \lambda_{1} s\right)} \frac{\cos \left(e \chi_{2} s\right)}{\sin \left(e \lambda_{2} s\right)}-1\right]
$$

It should be noted that $s \rightarrow i s$ is permissible provided there are no singularities associated with pair creation. Separating the divergent terms in the above expression we have

$$
\begin{aligned}
\mathcal{L}_{e f f}[F, H]=-(1+ & \left.e^{2} C_{e}\right) \mathcal{F}-\left(1-g^{2} C_{g}\right) \mathcal{H} \\
& -\frac{1}{8 \pi^{2}} \int_{0}^{\infty} \frac{d s}{s^{3}} e^{-m^{2} s}\left[\left(e \lambda_{1} s\right)\left(e \lambda_{2} s\right) \frac{\cos \left(e \chi_{1} s\right)}{\sin \left(e \lambda_{1} s\right)} \frac{\cos \left(e \chi_{2} s\right)}{\sin \left(e \lambda_{2} s\right)}-1-\frac{2}{3} e^{2} s^{2} \mathcal{F}+g^{2} s^{2} \mathcal{H}\right]
\end{aligned}
$$

where

$$
C_{e}=\frac{1}{12 \pi^{2}} \int_{0}^{\infty} \frac{d s}{s} e^{-m^{2} s} \quad \text { and } \quad C_{g}=\frac{1}{8 \pi^{2}} \int_{0}^{\infty} \frac{d s}{s} e^{-m^{2} s}
$$


We can absorb the divergences $C_{e}$ and $C_{g}$ by suitable change of scale of the fields and couplings. Identifying the quantities used till now by the subscript zero, we define the following scale transformations

$$
\begin{aligned}
& e^{2}=\frac{e_{0}^{2}}{1+e_{0}^{2} C_{e}} \quad \mathcal{F}=\left(1+e_{0}^{2} C_{e}\right) \mathcal{F}_{0} \quad \mathcal{G}=\left(1+e_{0}^{2} C_{e}\right) \mathcal{G}_{0} \\
& g^{2}=\frac{g_{0}^{2}}{1-g_{0}^{2} C_{g}} \quad \mathcal{H}=\left(1-g_{0}^{2} C_{g}\right) \mathcal{H}_{0} \quad \mathcal{I}^{2}=\left(1+e_{0}^{2} C_{e}\right)\left(1-g_{0}^{2} C_{g}\right) \mathcal{I}_{0}^{2}
\end{aligned}
$$

Using the above scale transformations we obtain the divergence free effective Lagrangian to be

$\mathcal{L}_{e f f}[F, H]=-\mathcal{F}-\mathcal{H}-\frac{1}{8 \pi^{2}} \int_{0}^{\infty} \frac{d s}{s^{3}} e^{-m^{2} s}\left[\left(e \lambda_{1} s\right)\left(e \lambda_{2} s\right) \frac{\cos \left(e \chi_{1} s\right)}{\sin \left(e \lambda_{1} s\right)} \frac{\cos \left(e \chi_{2} s\right)}{\sin \left(e \lambda_{2} s\right)}-1-\frac{2}{3} e^{2} s^{2} \mathcal{F}+g^{2} s^{2} \mathcal{H}\right]$

This is the generalisation of the Euler-Heisenberg lagrangian to include constant Maxwell and Kalb-Ramond fields. Explicitly expanding the above expression for weak fields using the series expansions for $\cos x$ and $(\sin x)^{-1}$ we get

$$
\mathcal{L}_{\text {eff }}[F, H]=-\mathcal{F}-\mathcal{H}+\frac{1}{8 \pi^{2}} \frac{1}{m^{4}}\left\{\frac{4}{45} e^{4} \mathcal{F}^{2}+\frac{7}{45} e^{4} \mathcal{G}^{2}-\frac{1}{6} g^{4} \mathcal{H}^{2}+2 e^{2} g^{2} \mathcal{I}^{2}\right\}+\ldots
$$

In addition to the self-interaction term $-\frac{1}{6} g^{4} \mathcal{H}^{2}$ for the Kalb-Ramond fields an interaction term between the Maxwell field and the Kalb-Ramond field given by $2 e^{2} g^{2} \mathcal{I}^{2}$ is also induced in the leading order. Observe that for the case of $g \rightarrow 0$ we get back Schwinger's result. It is interesting to note that the sign of the $\mathcal{H}^{2}$ term is negative.

\section{ACKNOWLEDGEMENTS}

K. V. Shajesh would like to express his appreciation to The Institute of Mathematical Sciences for his visit during which this work was undertaken.

[1] W. Heisenberg and H. Euler, Z. Physik 98, 714 (1936).

[2] V. Weisskopf, Kgl. Danske Videnskab. Selskabs. Mat. - Fys. Med. 14, No. 6 (1936). 
[3] J. Schwinger, Phys. Rev. 82664 (1951).

[4] C. Teitelboim, Phys. Lett. B 167, 63, 69 (1986).

[5] M. Henneaux and C. Teitelboim, Foundations of Physics 16, 593 (1986).

[6] Y. Nambu, Phys. Rep. 23 C, 250 (1976).

[7] M. Kalb and P. Ramond, Phys. Rev. D 9, 2273 (1974).

[8] M. Green, J. Schwarz and E. Witten, Superstring Theory, Vol I and II, Cambridge (1985).

[9] H. Goldstein, Classical Mechanics 2nd ed. eqn. 9.14 (1980).

[10] V. Fock, Physik. Z. Sowjetunion 12, 404 (1937).

[11] Y. Nambu, Prog. Theor. Phys. 5, 82 (1950).

[12] B. S. DeWitt, Phys. Rep. 19, 295 (1975).

[13] A. H. Taub, Phys. Rev. 73, 786 (1948). 